\title{
ANALYTICAL SOLUTIONS OF SOME STEADY-STATE ELECTRICAL PROBLEMS IN THE RECTANGULAR DOMAIN
}

\author{
Masayuki OKABE \\ Mitsui Mining \& Smelting Company, Ltd., Nihnbashi-Muromachi, Chuo-Ku, Tokyo 103, Japan \\ Noboru KIKUCHI \\ Department of Mechanical Engineering and Applied Mechanics, The University of Michigan, \\ Ann Arbor, MI 48109, U.S.A. \\ Received 21 July 1981 \\ Revised manuscript received 24 May 1982
}

\begin{abstract}
Analytical solutions for the electrical potential are developed within a rectangular domain under dirichlet and Neumann boundary conditions. Examples enable us to examine currently used numerical techniques. All solutions are applicable to the houndary element method as typical fundamental solutions.
\end{abstract}

\section{Introduction}

In potential theory, the boundary element method is recognized as a powerful numerical tool. Usually it works with the simple fundamental solution based on the whole- or half-space scalar Green function. In the case of a uniform and isotropic domain connected only to isolated regions, a Fredholm integral equation of the second kind with respect to the potential can be derived $[1,2]$ which is solved numerically by using piecewise polynomial trial functions [3-7] kept over every boundary elements. As is presented in this paper, if we practice numerical quadratures in computing the integral equation, then unacceptable results are obtained within the vicinity of the boundary surface. In the case of analytical integrations, on the other hand, we have highly accurate boundary-element solutions everywhere. However, analytical integrations are not always possible, and hence more complicated fundamental solutions should be considered in the boundary integral approach.

This paper is devoted to the analytical solutions of electrical potential within the simple rectangular domain. We utilize the image method in conjunction with a conformal mapping, and the solutions are given in the form of infinite series. Examples are then presented under Dirichlet and Neumann boundary conditions.

Through the weighted residual formulations, we further derive a general integral equation. Here attachment of the positioning constant to the governing equation is of great significance [8]. Under the Neumann boundary condition, we have the Fredholm integral equation of the second kind with respect to the potential. The problem is then solved numerically based on the whole-space scalar Green function. Typical finite-element solutions are also compared to exact values. 


\section{General infinite fissure problem}

The problem we first consider is the two-dimensional infinite fissure $\Omega$ of uniform and isotropic conductivity $\sigma$ embedded into the universal whole-space $\Omega_{0}$ of conductivity $\sigma_{0}$ (Fig. 1). Two walls $\Gamma_{h}^{+}$and $\Gamma_{h}^{-}$lie at $y=h$ and $y=-h$, respectively. A dc point source of current intensity $I$ is placed at $\boldsymbol{x}_{s}^{+}=\left(x_{s}^{+}, y_{s}^{+}\right)$with another source of $-I$ at $\boldsymbol{x}_{s}^{-}=\left(x_{s}^{-}, y_{s}^{-}\right)$both within $\Omega$.

The electrical potential at $x=(x, y)$ in $\Omega$ denoted by $\phi(x)$ is governed by

$$
\sigma \nabla^{2} \phi(x)+I \delta\left(x-x_{s}^{+}\right)-I \delta\left(x-x_{s}^{-}\right)=0, \quad x \in \Omega,
$$

while the governing equation for the potential $\phi_{0}(x)$ in $\Omega_{0}$ can be written as

$$
\sigma_{0} \nabla^{2} \phi_{0}(x)=0, \quad y \leq-h \text { or } h \leq y .
$$

Here $\nabla^{2}$ denotes the Laplacian with respect to $x$, and $\delta$ is the Dirac distribution.

The boundary conditions that apply to this problem are

and

$$
\phi(x)=\phi_{0}(x), \quad y= \pm h,
$$

$$
\sigma \partial \phi(x) / \partial y=\sigma_{0} \partial \phi_{0}(x) / \partial y, \quad y= \pm h .
$$

The natural Dirichlet boundary conditions at infinity should further be satisfied such that

and

$$
\phi(x) \rightarrow 0, \quad\left\|x-x_{s}^{+}\right\| \rightarrow \infty \text { and }\left\|x-x_{s}^{-}\right\| \rightarrow \infty,
$$

$$
\phi_{0}(x) \rightarrow 0, \quad\left\|x-x_{s}^{+}\right\| \rightarrow \infty \quad \text { and } \quad\left\|x-x_{s}^{-}\right\| \rightarrow \infty .
$$

Here $\|\cdot\|$ designates the usual norm in $(x, y)$.

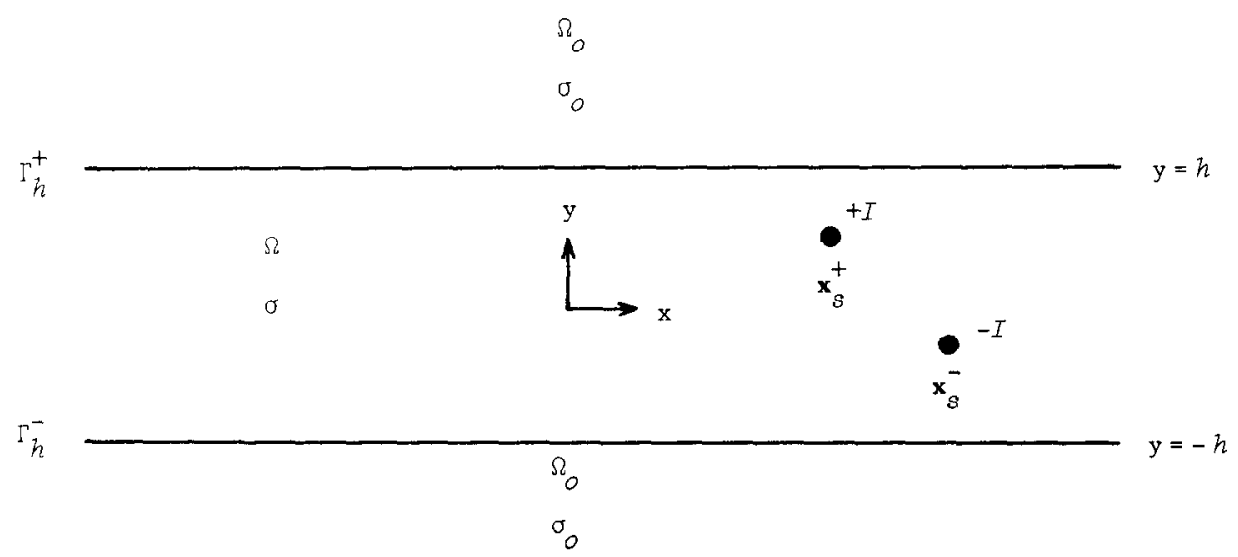

Fig. 1. Geometry of the infinite fissure problem. The infinite fissure $\Omega$ of conductivity $\sigma$ is embedded into otherwise uniform and isotropic whole-space $\Omega_{0}$ of conductivity $\sigma_{0}$. The boundary interfaces $\Gamma_{h}^{+}$and $\Gamma_{h}^{-}$are expressed as $y=h$ and $y=-h$, respectively. 
THEOREM 2.1. For the general infinite fissure problem, the potential $\phi(x)$ in $\Omega$ can be written as

where

$$
\phi(x)=\frac{I}{2 \pi \sigma} \sum_{n=-\infty}^{\infty} \kappa^{n} \ln \left[R_{n}\left(x, x_{s}^{-}\right) / R_{N}\left(x, x_{s}^{+}\right)\right], \quad-h \leq y \leq h,
$$

and

$$
R_{n}\left(\boldsymbol{x}, \boldsymbol{x}_{s}\right)=\left[\left(x-x_{s}\right)^{2}+\left\{y-(-1)^{n} y_{\mathrm{s}}-2 n h\right\}^{2}\right]^{1 / 2},
$$

$$
\kappa=\left(\sigma-\sigma_{0}\right) /\left(\sigma+\sigma_{0}\right) .
$$

The infinite summation is taken with respect to the integer $n$.

The potential $\phi_{0}(x)$ in $\Omega_{0}$ too can be expressed in an infinite series form such that

$$
\phi_{0}(x)=\frac{(1+\kappa) I}{2 \pi \sigma} \sum_{n=0}^{\infty} \kappa^{n} \ln \left[R_{n}\left(x, x_{\mathrm{s}}^{-}\right) / R_{n}\left(x, x_{\mathrm{s}}^{+}\right)\right], \quad y \leq-h,
$$

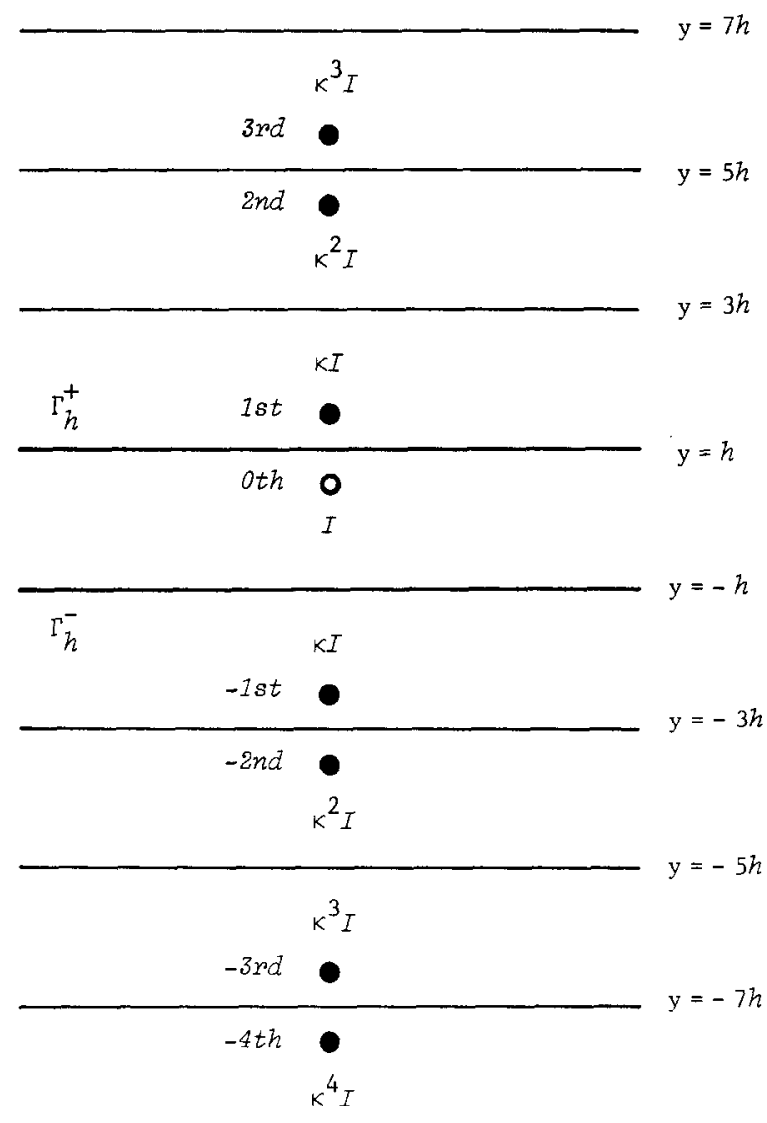

Fig. 2. Placement of the image sources associated with solutions within the infinite fissure $\Omega$. Only the original source should be placed within $\Omega$.

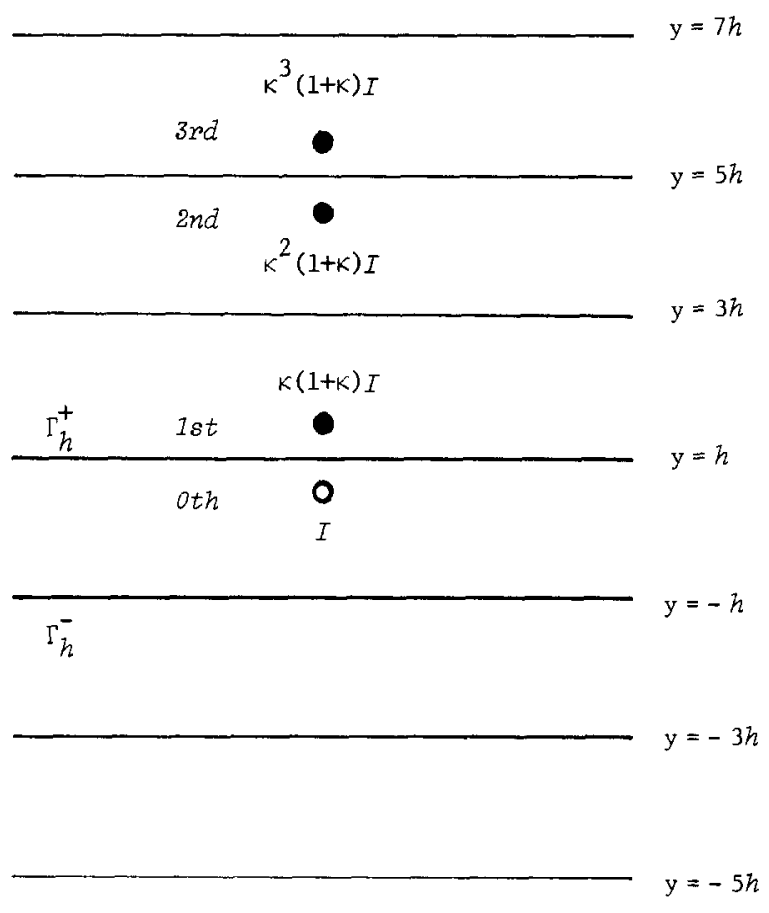

Fig. 3. Placement of the image sources associated with solutions in the outside domain $\Omega_{0}$ with $y \leq-h$, where the primary source is placed within the infinite fissure $\Omega$. No image sources should be placed within $\Omega_{0}$ of $y \leq-h$ to avoid unreal singularities. 
and

$$
\phi_{0}(x)=\frac{(1+\kappa) I}{2 \pi \sigma} \sum_{n=-\infty}^{0} \kappa^{n} \ln \left[R_{n}\left(x, x_{\mathrm{s}}^{-}\right) / R_{n}\left(x, x_{\mathrm{s}}^{+}\right)\right], \quad h \leq y .
$$

PROOF. In the simple whole-space of conductivity $\sigma$, let us place $n$th sources of current intensity $\kappa^{n} I$ and $-\kappa^{n} I$ at $\left(x_{\mathrm{s}}^{+},(-1)^{n} y_{\mathrm{s}}^{+}+2 n h\right)$ and $\left(x_{\mathrm{s}}^{-},(-1)^{n} y_{\mathrm{s}}^{-}+2 n h\right)$, respectively (Fig. 2). Then we have (7).

Let $n$ be nonnegative. Then evidently $(n+1)$ st sources are the images of $(-n)$ th sources with respect to $\Gamma_{h}^{+}$, while $(-n-1)$ st sources are those of $n$th sources reflected in $\Gamma_{h}^{-}$.

Let $n$th sources be of current intensity $\kappa^{n}(1+\kappa) I$ and $-\kappa^{n}(1+\kappa) I$ for nonnegative $n$, and let $n$th sources with negative $n$ be extinguished (Fig. 3). Then we have (10). Obviously $n$th sources related to $\phi_{0}(x)$ of $(10)$ and $n$th and $(-n-1)$ st sources associated with $\phi(x)$ of $(7)$ realize the continuity of the potential as well as that of the normal current density across $\Gamma_{h}^{-}$. Hence the boundary conditions (3) and (4) are satisfied on $\Gamma_{h}^{-}$of $y=-h$.

In the same manner, we can prove that $\phi(x)$ of (7) and $\phi_{0}(x)$ of (11) satisfy (3) and (4) on $\Gamma_{h}^{+}$ of $y=h$.

\section{Infinite fissure problem under the Dirichlet boundary condition}

Consider the perfectly conductive domain $\Omega_{0}$ with $\sigma_{0}=\infty$. Then we have

$$
\phi_{0}(x)=0, y \leq-h \text { and } h \leq y
$$

Noting (3), our problem is thus to solve the differential equation (1) under the Dirichlet boundary condition of the form

$$
\phi(x)=0, \quad y= \pm h
$$

Evidently the natural boundary condition (5) at infinity should also be satisfied.

THEOREM 3.1. In the Dirichlet infinite fissure problem, the electrical potential $\boldsymbol{\phi}(\boldsymbol{x})$ can be expressed as

$$
\phi(x)=\frac{I}{4 \pi \sigma} \ln \left[F\left(x, y, x_{\mathrm{s}}^{-}, y_{\mathrm{s}}^{-}, h\right) G\left(x, y, x_{\mathrm{s}}^{+}, y_{\mathrm{s}}^{+}, h\right) / F\left(x, y, x_{\mathrm{s}}^{+}, y_{\mathrm{s}}^{+}, h\right) G\left(x, y, x_{\mathrm{s}}^{-}, y_{\mathrm{s}}^{-}, h\right)\right]
$$

where

$$
F\left(x, y, x_{\mathrm{s}}, y_{\mathrm{s}}, h\right)=\mathrm{e}^{\pi x / h}+\mathrm{e}^{\pi x_{\mathrm{s}} / h}-2 \mathrm{e}^{\pi\left(x+x_{\mathrm{s}}\right) / 2 h} \cos \pi\left(y-y_{\mathrm{s}}\right) / 2 h,
$$

and

$$
G\left(x, y, x_{\mathrm{s}}, y_{\mathrm{s}}, h\right)=\mathrm{e}^{\pi x / h}+\mathrm{e}^{\pi x_{\mathrm{s}} / h}+2 \mathrm{e}^{\pi\left(x+x_{\mathrm{s}}\right) / 2 h} \cos \pi\left(y+y_{\mathrm{s}}\right) / 2 h .
$$

PROOF. Let us introduce the conformal mapping of the form

$$
\pi(x+\mathrm{i} y)=2 h \ln (X+\mathrm{i} Y)
$$


where i designates the imaginary unit. Then the infinite fissure $\Omega$ of Fig. 1 with $-h \leq y \leq h$ can conformally be transformed into the half-space of Fig. 4 with $X \geq 0$ [9].

Let $\boldsymbol{X}_{\mathrm{s}}^{+}=\left(X_{\mathrm{s}}^{+}, Y_{\mathrm{s}}^{+}\right)$and $\boldsymbol{X}_{\mathrm{s}}^{-}=\left(X_{\mathrm{s}}^{-}, Y_{\mathrm{s}}^{-}\right)$denote the transformed source points related to $\boldsymbol{X}_{\mathrm{s}}^{+}$ and $\boldsymbol{x}_{\mathrm{s}}^{-}$, respectively $\left(X_{\mathrm{s}}^{+}>0\right.$ and $\left.X_{\mathrm{s}}^{-}>0\right)$. The potential at $X=(X, Y)$ associated with that $\boldsymbol{x}$ can then be given as

$$
\phi(X)=\frac{I}{2 \pi \sigma}\left\{\ln \left(\left\|\boldsymbol{X}-\boldsymbol{X}_{\mathrm{s}}^{-}\right\| /\left\|\boldsymbol{X}-\boldsymbol{X}_{\mathrm{s}}^{+}\right\|\right)+\kappa \ln \left(\left\|\tilde{\boldsymbol{X}}-\boldsymbol{X}_{\mathrm{s}}^{-}\right\| /\left\|\tilde{\boldsymbol{X}}-\boldsymbol{X}_{\mathrm{s}}^{+}\right\|\right)\right\},
$$

for $X \geq 0$. Here $\tilde{\boldsymbol{X}}$ denotes the image point of $\boldsymbol{X}$ reflected in the plane of $X=0$, i.e., $\tilde{\boldsymbol{X}}=(-X, Y)$, and $\kappa$ is given by (9). In this case, $\kappa=-1$ since $\sigma_{0}=\infty$.

The conformal mapping of (17) can be solved as

$$
X=\mathrm{e}^{\pi x / h} \cos \pi y / 2 h \quad \text { and } \quad Y=\mathrm{e}^{\pi x / h} \sin \pi y / 2 h, \quad-h \leq y \leq h .
$$

Substituting (19) with respect to $\boldsymbol{X}, \boldsymbol{X}_{\mathrm{s}}^{+}$and $\boldsymbol{X}_{\mathrm{s}}^{-}$into (18), we have (14).

REMARK 3.2. Corresponding to (1), the Poisson's equation in $(X, Y)$ can be written as

$$
\sigma \nabla^{2} \phi(X)+I \delta\left(X-X_{\mathrm{s}}^{+}\right)-I \delta\left(X-X_{s}^{-}\right)=0, \quad X \geq 0 .
$$

Here $\nabla^{2}$ is related to $\boldsymbol{X}$. Notice that the Jacobian never appears in the transformed governing equation.

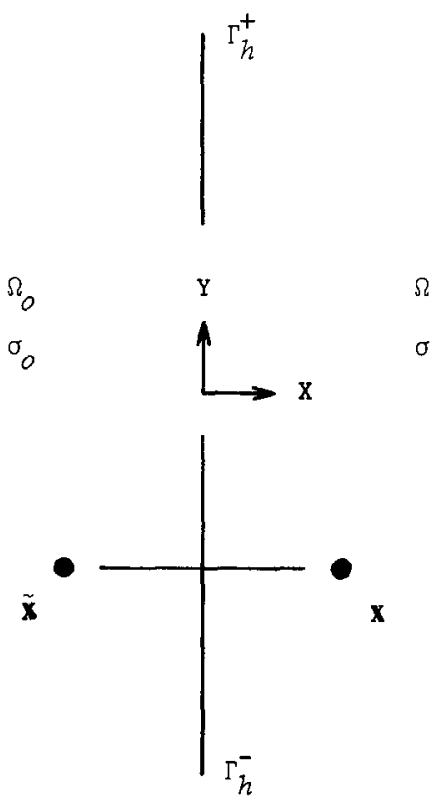

Fig. 4. Transformed space ( $X, Y)$ after the conformal mapping of (17). The half-space with positive $X$ corresponds to the infinite fissure $\Omega$ in $(x, y)$. The image of $X=(X, Y)$ reflected in the plane of $X=0$ is denoted by $\tilde{X}=(-X, Y)$. 
THEOREM 3.3. The potential of (14) can alternately be expressed as

$$
\phi(x)=\frac{I}{2 \pi \sigma} \sum_{n=-\infty}^{\infty}(-1)^{n} \ln \left[R_{n}\left(x, x_{\mathrm{s}}^{-}\right) / R_{n}\left(x, x_{\mathrm{s}}^{+}\right)\right] .
$$

Here $R_{n}$ is given by (8).

\section{Infinite fissure problem under the Neumann boundary condition}

In the general fissure problem, let the conductivity $\sigma_{0}$ of the universal domain $\Omega_{0}$ be zero. Then on the walls $\Gamma_{h}^{+}$and $\Gamma_{h}^{-}$, the potential $\phi(x)$ should satisfy the typical Neumann boundary condition of the form

$$
\partial \phi(x) / \partial y=0, \quad y= \pm h .
$$

Evidently the natural boundary condition (5) at infinity should further be satisfied with the Poisson's equation (1).

Notice that the potential $\phi_{0}(x)$ has no physical meaning in this case.

THEOREM 4.1 In the Neumann infinite fissure problem, the electrical potential $\phi(x)$ can be expressed as

$$
\phi(x)=\frac{I}{4 \pi \sigma} \ln \left[F\left(x, y, x_{\mathrm{s}}^{-}, y_{\mathrm{s}}^{-}, h\right) G\left(x, y, x_{\mathrm{s}}^{-}, y_{\mathrm{s}}^{-}, h\right) / F\left(x, y, x_{\mathrm{s}}^{+}, y_{\mathrm{s}}^{+}, h\right) G\left(x, y, x_{\mathrm{s}}^{+}, y_{\mathrm{s}}^{+}, h\right)\right] .
$$

Here $F$ and $G$ are given by (15) and (16), respectively.

PROOF. The conformal mapping of (17) is valid also in this case, and hence the potential $\phi(X)$ at $\boldsymbol{X}$ after transformation is given by (18) but with $\kappa=1$.

THEOREM 4.2. The potential of (23) can be rewritten in an infinite series form as

$$
\phi(x)=\frac{I}{2 \pi \sigma} \sum_{n=-\infty}^{\infty} \ln \left[R_{n}\left(x, x_{\mathrm{s}}^{-}\right) / R_{n}\left(x, x_{\mathrm{s}}^{+}\right)\right]
$$

Here $R_{n}$ is given by (8).

REMARK 4.3. Although the potential $\phi_{0}(x)$ has no physical meaning, the solutions $\phi(x)$ of (7) in the general infinite fissure problem produce those of the Neumann problem after substitution of $\kappa=1$. This feature is of significance in the boundary element approach.

REMARK 4.4. The conformal mapping of (17) is inadequate if $-1<\kappa<1(\kappa \neq 0)$. 


\section{Rectangle problem under the Dirichlet boundary condition}

We now consider a rectangular domain portrayed in Fig. 5 with uniform and isotropic conductivity $\sigma$ embedded into otherwise perfectly conductive whole-space. A dc point source of current intensity $I$ is placed at $x_{\mathrm{s}}$ within $\Omega$. Obviously the potential $\phi(x)$ is to be zero on $\Gamma_{h}^{+}$ of $y=h, \Gamma_{h}^{-}$of $y=h, \Gamma_{g}^{+}$of $x=g$ and $\Gamma_{g}^{-}$of $x=g$ such that

$$
\phi(x)=0, \quad x= \pm g \quad \text { or } \quad y= \pm h .
$$

The governing equation is written as

$$
\sigma \nabla^{2} \phi(x)+I \delta\left(x-x_{\mathrm{s}}\right)=0, \quad x \in \Omega .
$$

THEOREM 5.1. In the Dirichlet rectangle problem, the electrical potential can be written as

$$
\phi(\boldsymbol{x})=\frac{I}{4 \pi \sigma} \sum_{m=-\infty}^{\infty}(-1)^{m} \ln \left[G\left(x, y, x_{\mathrm{s}}^{m}, y_{\mathrm{s}}, h\right) / F\left(x, y, x_{\mathrm{s}}^{m}, y_{\mathrm{s}}, h\right)\right],
$$

where

$$
x_{\mathrm{s}}^{m}=(-1)^{m} x_{\mathrm{s}}+2 m g .
$$

Here the infinite summation is taken with respect to the integer $m$, and $F$ and $G$ are given by (15) and (16), respectively.

$P R O O F$. We apply the image procedure with $\kappa=-1$ in Theorem 2.1 with respect to $\Gamma_{g}^{+}$and $\Gamma_{\mathrm{s}}^{-}$. Then our problem can be solved as the Dirichlet infinite fissure problem with a series of sources of current intensity $(-1)^{m} I$ placed at $\left(x_{\mathrm{s}}^{m}, y_{\mathrm{s}}\right)$.

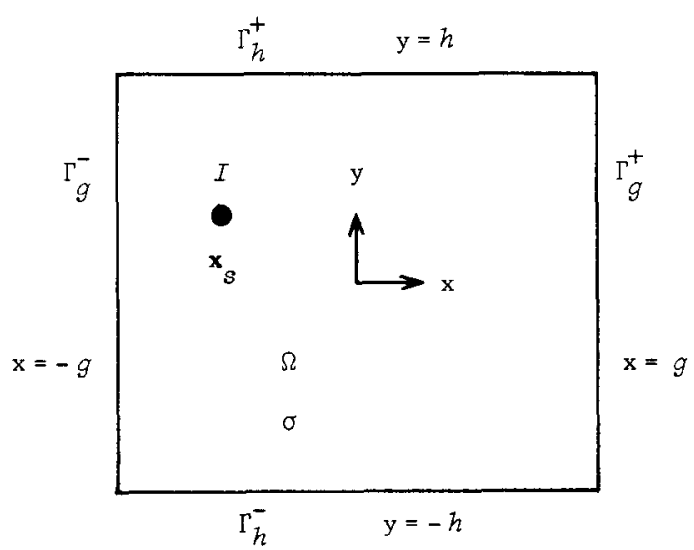

Fig. 5. Rectangular domain $\Omega$ with uniform and isotropic conductivity $\sigma$. A dc point source of current intensity $I$ is placed at $x_{\mathrm{s}}$ within $\Omega$. The walls are expressed as $x= \pm g$ and $y= \pm h$. 
THEOREM 5.2. The potential of (27) can be rewritten as

$$
\phi(x)=\frac{-I}{4 \pi \sigma} \sum_{m=-\infty}^{\infty} \sum_{n=-\infty}^{\infty}(-1)^{m+n} \ln \left[\left(x-x_{\mathrm{s}}^{m}\right)^{2}+\left(y-y_{\mathrm{s}}^{n}\right)^{2}\right],
$$

where

$$
y_{\mathrm{s}}^{n}=(-1)^{n} y_{\mathrm{s}}+2 n h .
$$

PROOF. In the simple whole-space of conductivity $\sigma$, we place the image source of intensity $(-1)^{m+n} I$ at $\left(x_{\mathrm{s}}^{m}, y_{\mathrm{s}}^{n}\right)$. The image procedure in two directions (fig. 6) thus yields (29).

THEOREM 5.3. Let $J_{x}(x)$ and $J_{y}(x)$ denote the current densities at $x$ in the $x$-and $y$-directions, respectively. Then in the Dirichlet rectangle problem, we have

and

$$
J_{x}(x)=\frac{1}{4} I \sum_{m=-\infty}^{\infty}(-1)^{m}\left\{F_{x}\left(x, y, x_{\mathrm{s}}^{m}, y_{\mathrm{s}}, h\right)-G_{x}\left(x, y, x_{\mathrm{s}}^{m}, y_{\mathrm{s}}, h\right)\right\},
$$

$$
J_{y}(x)=\frac{1}{4} I \sum_{m=-\infty}^{\infty}(-1)^{m}\left\{F_{y}\left(x, y, x_{\mathrm{s}}^{m}, y_{\mathrm{s}} h\right)-G_{y}\left(x, y, x_{\mathrm{s}}^{m}, y_{\mathrm{s}}, h\right)\right\}
$$

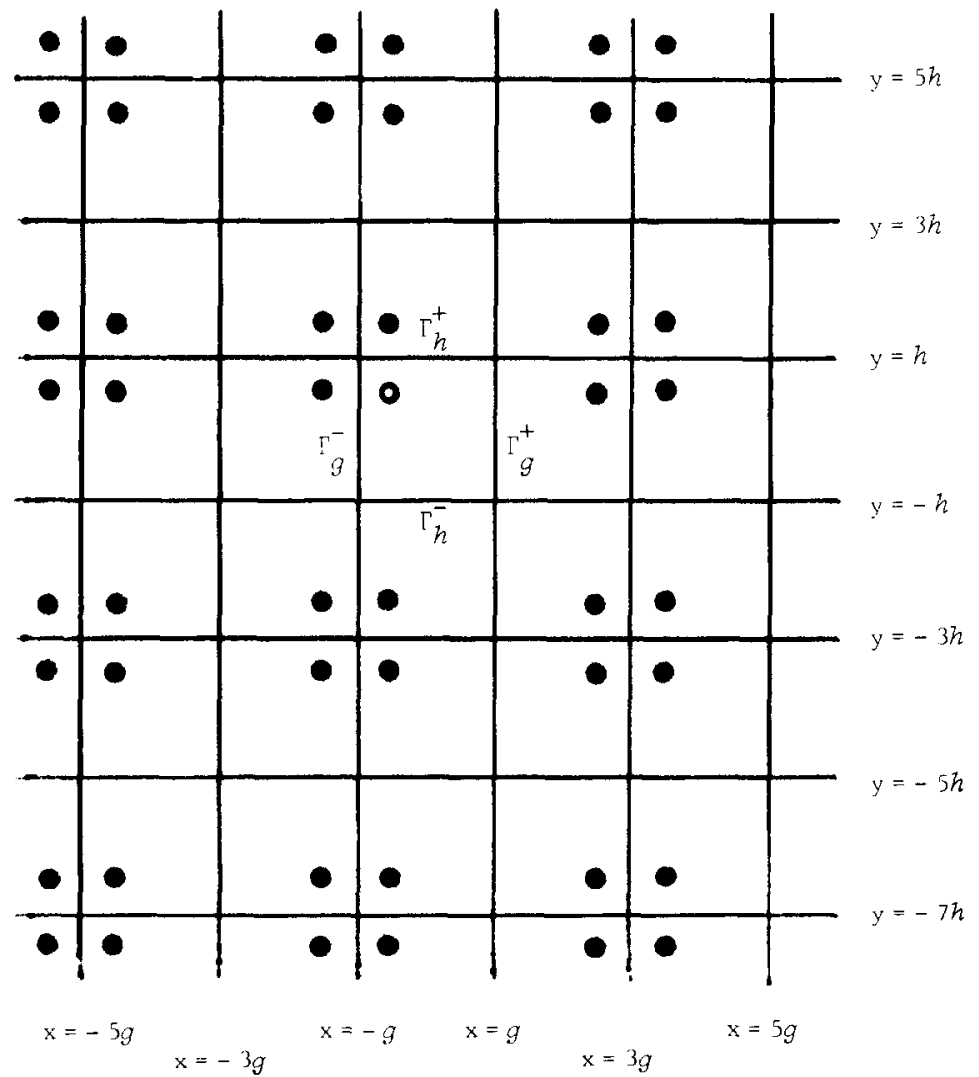

Fig. 6. Placement of the image sources in two directions associated with the rectangle $\Omega$ of Fig. 5 . The outside domain $\Omega_{0}$ should be perfectly conductive or isolated. 
Here $F_{x}, F_{y}, G_{x}$ and $G_{y}$ are given by

and

$$
\begin{aligned}
& F_{x}\left(x, y, x_{\mathrm{s}}, y_{\mathrm{s}}, h\right)=\left\{\mathrm{e}^{\pi x / h}-\mathrm{e}^{\pi\left(x+x_{\mathrm{s}}\right) / 2 h} \cos \pi\left(y-y_{\mathrm{s}}\right) / 2 h\right\} / h F\left(x, y, x_{\mathrm{s}}, y_{\mathrm{s}}, h\right), \\
& F_{y}\left(x, y, x_{\mathrm{s}}, y_{\mathrm{s}}, h\right)=\left\{\mathrm{e}^{\pi\left(x+x_{\mathrm{s}}\right) / 2 h} \sin \pi\left(y-y_{\mathrm{s}}\right) / 2 h\right\} / h F\left(x, y, x_{\mathrm{s}}, y_{\mathrm{s}}, h\right), \\
& G_{x}\left(x, y, x_{\mathrm{s}}, y_{\mathrm{s}}, h\right)=\left\{\mathrm{e}^{\pi x / h}+\mathrm{e}^{\pi\left(x+x_{\mathrm{s}}\right) / 2 h} \cos \pi\left(y+y_{\mathrm{s}} / 2 h\right\} / h G\left(x, y, x_{\mathrm{s}}, y_{\mathrm{s}}, h\right),\right.
\end{aligned}
$$

$$
G_{y}\left(x, y, x_{\mathrm{s}}, y_{\mathrm{s}}, h\right)=\left\{-\mathrm{e}^{\pi\left(x+x_{\mathrm{s}}\right) / 2 h} \sin \pi\left(y+y_{\mathrm{s}}\right) / 2 h\right\} / h G\left(x, y, x_{\mathrm{s}}, y_{\mathrm{s}}, h\right) .
$$

PROOF. The electric field is defined as $-\nabla \phi(x)$, where $\nabla$ is the Hamiltonian. Let $E_{x}(x)$ and $E_{y}(x)$ denote the electric field components at $x$ in the $x$-and $y$-directions, respectively. Then we have

$$
E_{x}(x)=-\partial \phi(x) / \partial x \text { and } E_{y}(x)=-\partial \phi(x) / \partial y .
$$

The Ohm's law further gives

$$
J_{x}(x)=\sigma E_{x}(x) \text { and } J_{y}(x)-\sigma E_{y}(x) .
$$

Thus differentiation of (27) gives (31) and (32). Notice that $\pi F_{x}$ represents $\partial(\ln F) / \partial x$.

\section{Rectangle problem under the Neumann boundary condition}

Consider next the rectangle $\Omega$ of Fig. 5 but embedded into otherwise isolated whole-space. Then on the walls, we have

and

$$
\partial \phi(x) / \partial x=0, \quad x= \pm g,
$$

$$
\partial \phi(x) / \partial y=0, \quad y= \pm h .
$$

Equations (36) and (37) state that no electric power diverges through the walls, and consequently a dc point source doesnot produce the steady state. We thus solve not (26) but (1) under the Neumann boundary conditions (36) and (37).

We define $\psi, \beta, K_{x}$ and $K_{y}$ by

and

$$
\begin{aligned}
& \psi\left(x, x_{\mathrm{s}}\right)=\sum_{m=-\infty}^{\infty} \ln \left[F\left(x, y, x_{\mathrm{s}}^{m}, y_{\mathrm{s}}, h\right) G\left(x, y, x_{\mathrm{s}}^{m}, y_{\mathrm{s}}, h\right)\right], \\
& \beta\left(\boldsymbol{x}, \boldsymbol{x}_{\mathrm{s}}\right)=\sum_{m=-\infty}^{\infty} \sum_{n=-\infty}^{\infty} \ln \left[\left(x-x_{\mathrm{s}}^{m}\right)^{2}+\left(y-y_{\mathrm{s}}^{n}\right)^{2}\right], \\
& K_{x}\left(\boldsymbol{x}, \boldsymbol{x}_{\mathrm{s}}\right)=\sum_{m=-\infty}^{\infty}\left\{F_{x}\left(x, y, x_{\mathrm{s}}^{m}, y_{\mathrm{s}}, h\right)+G_{x}\left(x, y, x_{\mathrm{s}}^{m}, y_{\mathrm{s}}, h\right)\right\},
\end{aligned}
$$

$$
K_{y}\left(\boldsymbol{x}, \boldsymbol{x}_{\mathrm{s}}\right)=\sum_{m=-\infty}^{\infty}\left\{F_{y}\left(x, y, x_{\mathrm{s}}^{m}, y_{\mathrm{s}}, h\right)+G_{y}\left(x, y, x_{\mathrm{s}}^{m}, y_{\mathrm{s}}, h\right)\right\} .
$$


THEOREM 6.1. In the Neumann rectangle problem, the electrical potential can be expressed as

$$
\phi(x)=\frac{I}{4 \pi \sigma}\left\{\psi\left(x, x_{\mathrm{s}}^{-}\right)-\psi\left(x, x_{\mathrm{s}}^{+}\right)\right\}+C_{1} .
$$

Ilere $C_{1}$ denotes an appropriate constant, and $\psi$ is given by (38).

PROOF. The image procedure in Theorem 5.1 is performed with $\kappa=1$. Then our problem can be solved as the Neumann infinite fissure problem with a series of sources of intensity $I$ placed at $\left(x_{\mathrm{s}}^{m}, y_{\mathrm{s}}\right)$. However, the natural boundary condition (5) at infinity is meaningless in the original Neumann rectangle problem, and consequently an appropriate constant $c_{1}$ is needed.

THEOREM 6.2. The potential of (42) can alternately be written as

$$
\phi(x)=\frac{I}{4 \pi \sigma}\left\{\beta\left(x, x_{\mathrm{s}}^{-}\right)-\beta\left(x, x_{\mathrm{s}}^{+}\right)\right\}+C_{2} .
$$

Here $C_{2}$ denotes an appropriate constant, and $\beta$ is given by (39).

THEOREM 6.3. In the Neumann rectangle problem, the current densities can be written as

and

$$
J_{x}(x)=\frac{1}{4} I\left\{K_{x}\left(x, x_{\mathrm{s}}^{+}\right)-K_{x}\left(x, x_{\mathrm{s}}^{-}\right)\right\},
$$

$$
J_{y}(x)=\frac{1}{4} I\left\{K_{y}\left(x, x_{\mathrm{s}}^{+}\right)-K_{y}\left(x, x_{\mathrm{s}}^{-}\right)\right\} .
$$

Here $K_{x}$ and $K_{y}$ are given by (40) and (41), respectively.

PROOF. Differentiation of (42) gives immediately (44) and (45). Evidently the constant $C_{1}$ disappears.

REMARK 6.4. The solutions of (42) to (45) in the Neumann rectangle problem allows point sources on the walls. In the Dirichlet rectangle problem, on the other hand, sources should not be placed on the boundaries.

REMARK 6.5. The image procedure in two directions of Fig. 6 is valid only in the case of $\kappa= \pm 1$ (or trivially $\kappa=0$ ).

REMARK 6.6. The image procedure is applicable also to the three-dimensional problems, but not the conformal mapping.

\section{Examples}

We can now examine currently used numerical techniques such as the finite element and boundary element methods. Examples we present are associated with a rectangle of $g=4$ and 


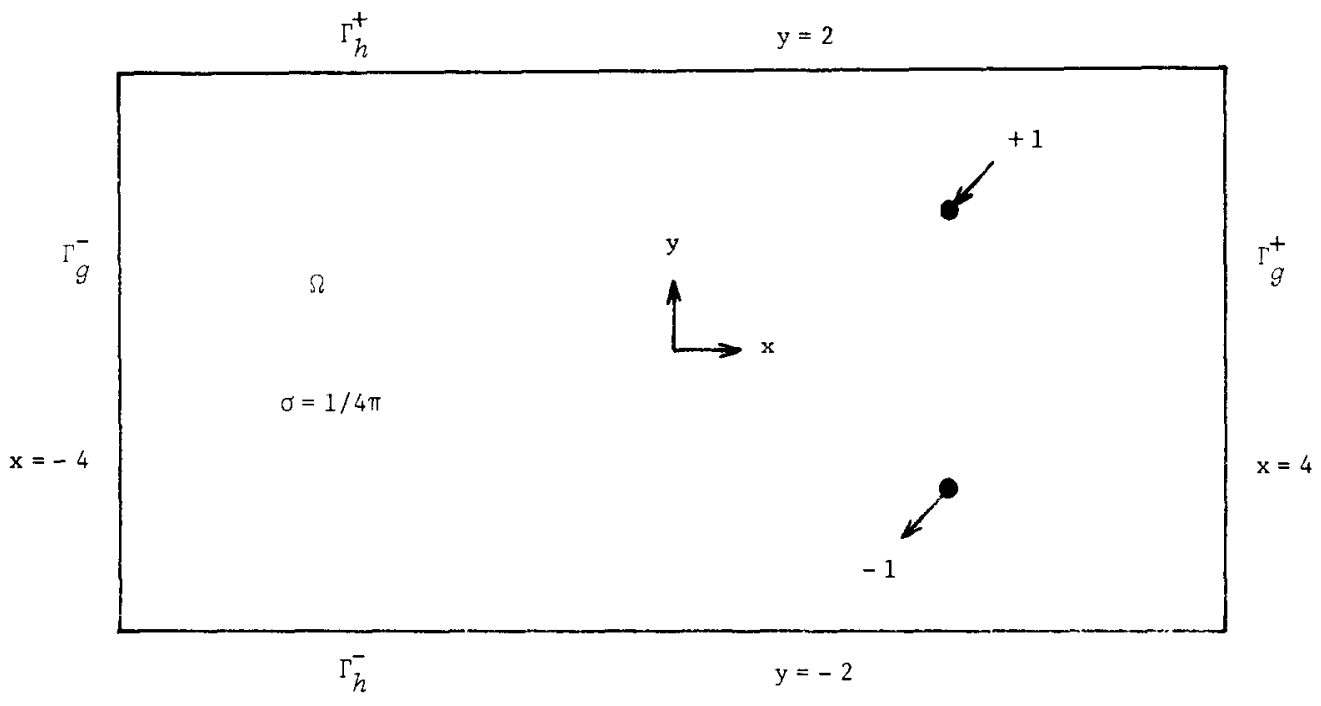

Fig. 7. Geometry of the targe examples with $g=4$ and $h=2$. The steady-state current of unit intensity is driven into the rectangle $\Omega$ of conductivity $\sigma=1 / 4 \pi$ at $(2,1)$ and removed from $(2,-1)$.

$h=2$ with conductivity $\sigma=1 / 4 \pi$ (Fig. 7). The steady-state current of unit intensity is driven into $\Omega$ at $(2,1)$ and removed from $(2,-1)$. In the Neumann rectangle problem, for simplicity, we put $C_{1}=0$ in (42).

We compute the potential and current densities by approximating the infinite series of $\Sigma_{-\infty}^{\infty}$ by the finite one of $\sum_{-N}^{N}$. Here $N$ is increased until the sufficient convergence is attained. In Tables 1 and 2 , the potential and current densities at $(0,1)$ due to the finite approximations are listed against $N$ in the Dirichlet and Neumann rectangle problems, respectively. All values converge quite rapidly.

At nodes regularly placed on a grid without vacancy, computed results are listed in Tables 3 and 4.

Table 1

Numerical solutions at $(0,1)$ under the finite approximations in the Dirichlet rectangle problem. The infinite series of $\sum_{-\infty}^{\infty}$ is approximated by the finite one of $\sum_{-N}^{N}$

\begin{tabular}{cccc}
\hline$N$ & $\phi(0,1)\left(\times 10^{-7}\right)$ & $J_{x}(0,1)\left(\times 10^{-8}\right)$ & $J_{y}(0,1)$ \\
\hline 0 & 1729635 & -2164740 & 0 \\
1 & 1726401 & -2160712 & 0 \\
2 & 1726401 & -2160712 & 0 \\
3 & 1726041 & -2160712 & 0 \\
4 & 1726401 & -2160712 & 0 \\
5 & 1726401 & -2160712 & 0 \\
\hline
\end{tabular}

Table 2

Solutions at $(0,1)$ under the finite approximations in the Neumann rectangle problem

\begin{tabular}{cccc}
\hline$N$ & $\phi(0,1)\left(\times 10^{-7}\right)$ & $J_{x}(0,1)\left(\times 10^{-8}\right)$ & $J_{y}(0,1)\left(\times 10^{-8}\right)$ \\
\hline 0 & 8438165 & -5431716 & -4981712 \\
1 & 8813035 & -5646611 & -5215981 \\
2 & 8813735 & -5646210 & -5216419 \\
3 & 8813736 & -5646210 & -5216419 \\
4 & 8813736 & -5646210 & -5216419 \\
5 & 8813736 & -5646210 & -5216419 \\
\hline
\end{tabular}




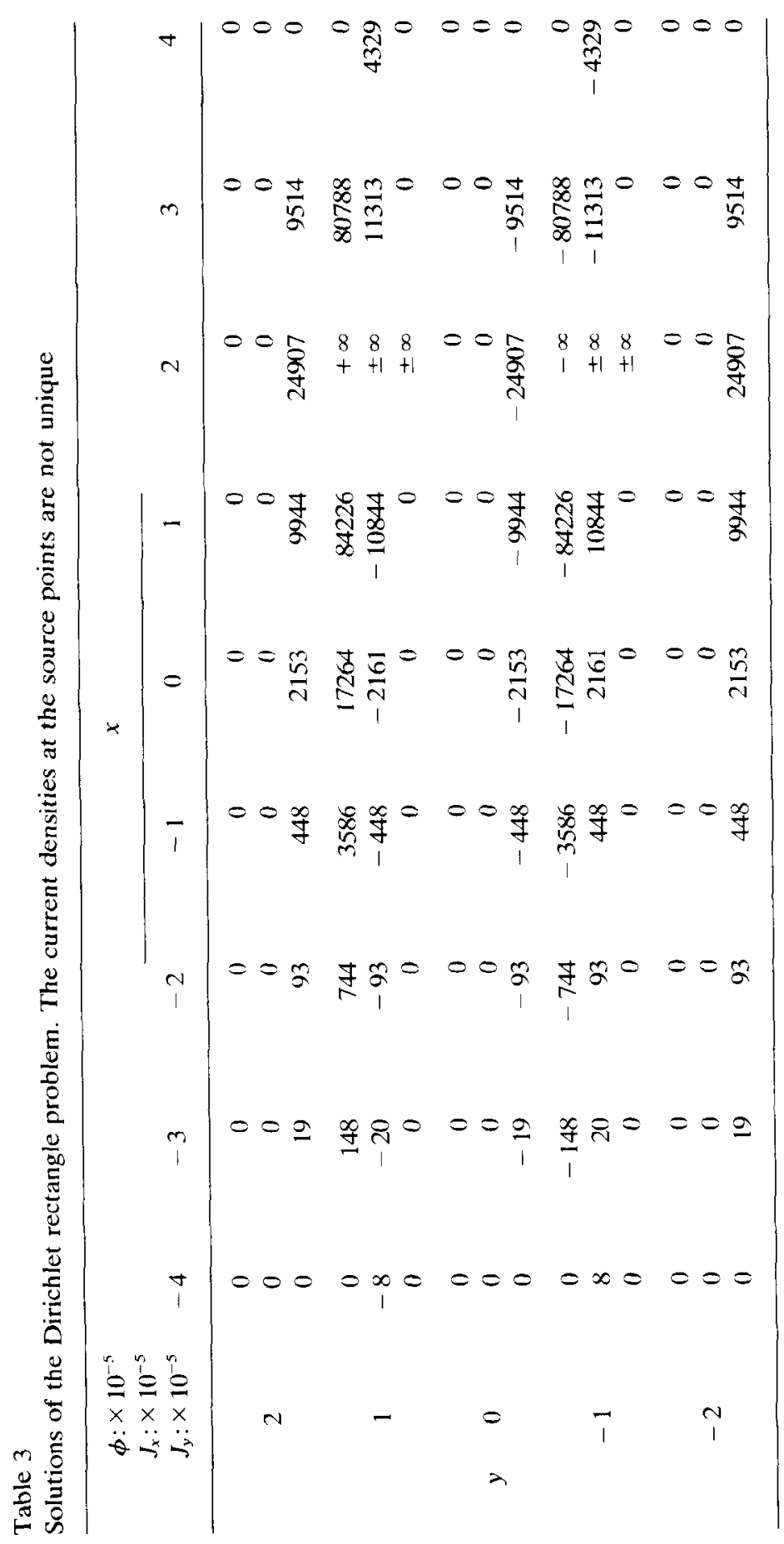




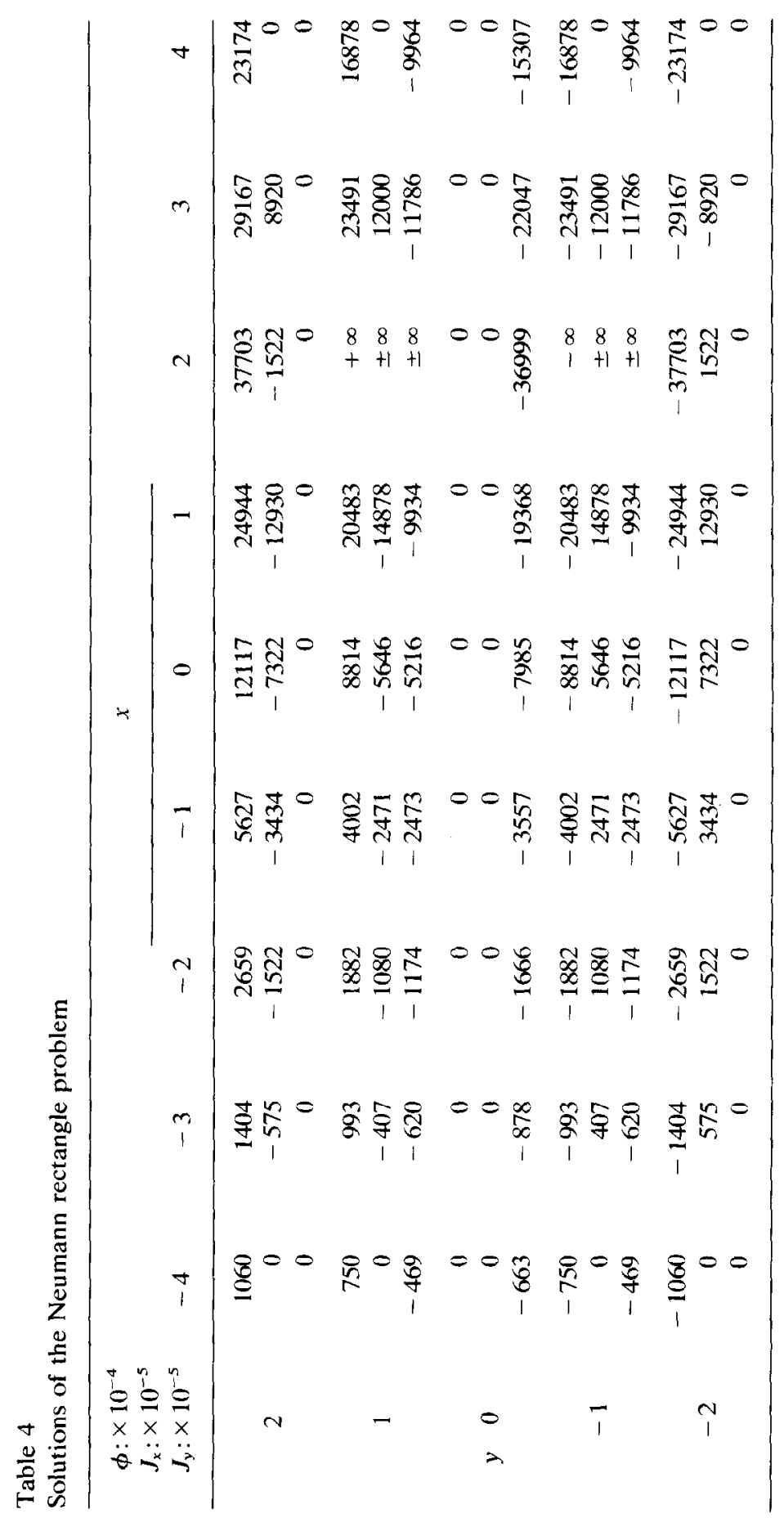


REMARK 7.1. In our examples, the following relations hold;

and

$$
\begin{aligned}
& \phi(x, y)=-\phi(x,-y), \\
& J_{x}(x, y)=-J_{x}(x,-y), \quad J_{y}(x, y)=J_{y}(x,-y),
\end{aligned}
$$

$$
\phi(x, 0)=J_{x}(x, 0)=0 .
$$

Notice that the potential $\phi$ and current density $J_{x}$ take strictly zero along the line of $y=0$.

\section{Boundary element applications}

Let $\phi^{*}(\tilde{\boldsymbol{x}}, \boldsymbol{x})$ be the fundamental solution governed by

$$
\sigma \tilde{\nabla}^{2} \phi^{*}(\tilde{x}, \boldsymbol{x})+\lambda(\boldsymbol{x}) \delta(\tilde{\boldsymbol{x}}-\boldsymbol{x})=0 .
$$

Here $\tilde{\nabla}^{2}$ is related to $\tilde{\boldsymbol{x}}=(\tilde{x}, \tilde{y})$, and $\lambda(x)$ is the positioning constant [8]. In the case of rectangular domain of Fig. $7, \lambda(x)$ takes $\frac{1}{4}$ if $x$ is placed at the vertex. For $x$ on the smooth edge and within $\Omega, \lambda(x)$ takes $\frac{1}{2}$ and 1 , respectively.

Noting (1) and (49), let us adopt the weighted residual of the form

$$
\begin{aligned}
0= & \int_{\Omega} \phi^{*}(\tilde{\boldsymbol{x}}, \boldsymbol{x})\left[\sigma \tilde{\nabla}^{2} \phi(\tilde{\boldsymbol{x}})+I \delta\left(\tilde{\boldsymbol{x}}-\boldsymbol{x}_{\mathrm{s}}^{+}\right)-I \delta\left(\tilde{\boldsymbol{x}}-\boldsymbol{x}_{\mathrm{s}}^{-}\right)\right] \mathrm{d} \Omega \\
& -\int_{\Omega} \phi(\tilde{\boldsymbol{x}})\left[\sigma \tilde{\nabla}^{2} \phi^{*}(\tilde{\boldsymbol{x}}, \boldsymbol{x})+\lambda(\boldsymbol{x}) \delta(\tilde{\boldsymbol{x}}-\boldsymbol{x})\right] \mathrm{d} \Omega .
\end{aligned}
$$

Here the differentiations and integrations are taken with respect to the field coordinates $\tilde{\boldsymbol{x}}$. then we have

$$
\lambda(x) \phi(x)=\phi_{\mathrm{P}}(x)-\int_{\Gamma} \sigma \phi(\tilde{\boldsymbol{x}}) \tilde{\boldsymbol{n}} \cdot \tilde{\nabla} \phi^{*}(\tilde{\boldsymbol{x}}, \boldsymbol{x}) \mathrm{d} \Gamma+\int_{\Gamma} \sigma \phi^{*}(\tilde{\boldsymbol{x}}, \boldsymbol{x}) \tilde{\boldsymbol{n}} \cdot \tilde{\nabla} \phi(\tilde{\boldsymbol{x}}) \mathrm{d} \Gamma,
$$

where $\tilde{\boldsymbol{n}}$ is the outward normal at $\tilde{\boldsymbol{x}}$ on $\Gamma$ from $\Omega, \tilde{\nabla}$ is the Hamiltonian related to $\tilde{\boldsymbol{x}}$ and $\phi_{\mathrm{P}}(\boldsymbol{x})$ denotes the primary potential deformed by

$$
\phi_{\mathrm{P}}(x)=I\left\{\phi^{*}\left(x_{\mathrm{s}}^{+}, x\right)-\phi^{*}\left(x_{\mathrm{s}}^{-}, x\right)\right\} .
$$

In the Neumann rectangle problem, (51) can be written as

$$
\lambda(x) \phi(x)=\phi_{\mathrm{P}}(x)-\int_{\Gamma} \sigma \phi(\tilde{\boldsymbol{x}}) \tilde{n} \cdot \bar{\nabla} \phi^{*}(\tilde{\boldsymbol{x}}, \boldsymbol{x}) \mathrm{d} \Gamma+C .
$$

Here $\hat{\Gamma}$ designates the part of $\Gamma$ where $\tilde{\boldsymbol{n}} \cdot \tilde{\nabla} \phi^{*}(\tilde{\boldsymbol{x}}, \boldsymbol{x}) \neq 0$, and $C$ denotes an appropriate constant. In the case of the whole-space scalar fundamental solution of the form 


$$
\phi^{*}(\tilde{x}, x)=-\frac{1}{2 \pi \sigma} \ln R_{0}(\tilde{x}, x)
$$

$\hat{\Gamma}$ is identical to $\Gamma$, i.e., $\hat{\Gamma}$ is composed of $\Gamma_{g}^{ \pm}$and $\Gamma_{h}^{ \pm}$. Here $R_{0}$ is given by (8). If we adopt another fundamental solution of the form

$$
\phi^{*}(\tilde{x}, x)=-\frac{1}{2 \pi \sigma} \ln \left[R_{0}(\tilde{x}, x) / R_{1}(\tilde{x}, x)\right]
$$

then we have

$$
\sigma \tilde{\boldsymbol{n}} \cdot \tilde{\nabla} \phi^{*}(\tilde{\boldsymbol{x}}, \boldsymbol{x})=0, \quad \tilde{\boldsymbol{x}} \in \Gamma_{h}^{+}
$$

and hence $\hat{\Gamma}$ is composed of $\Gamma_{g}^{ \pm}$and $\Gamma_{h}$.

In the boundary integral approach, the Fredholm integral equation (53) of the second kind is first to be solved on $x$ on $\Gamma$. We divide $\Gamma$ of Fig. 7 into 24 boundary elements of unit length, and on each boundary element the piecewise linear trial function is assumed for the potential.

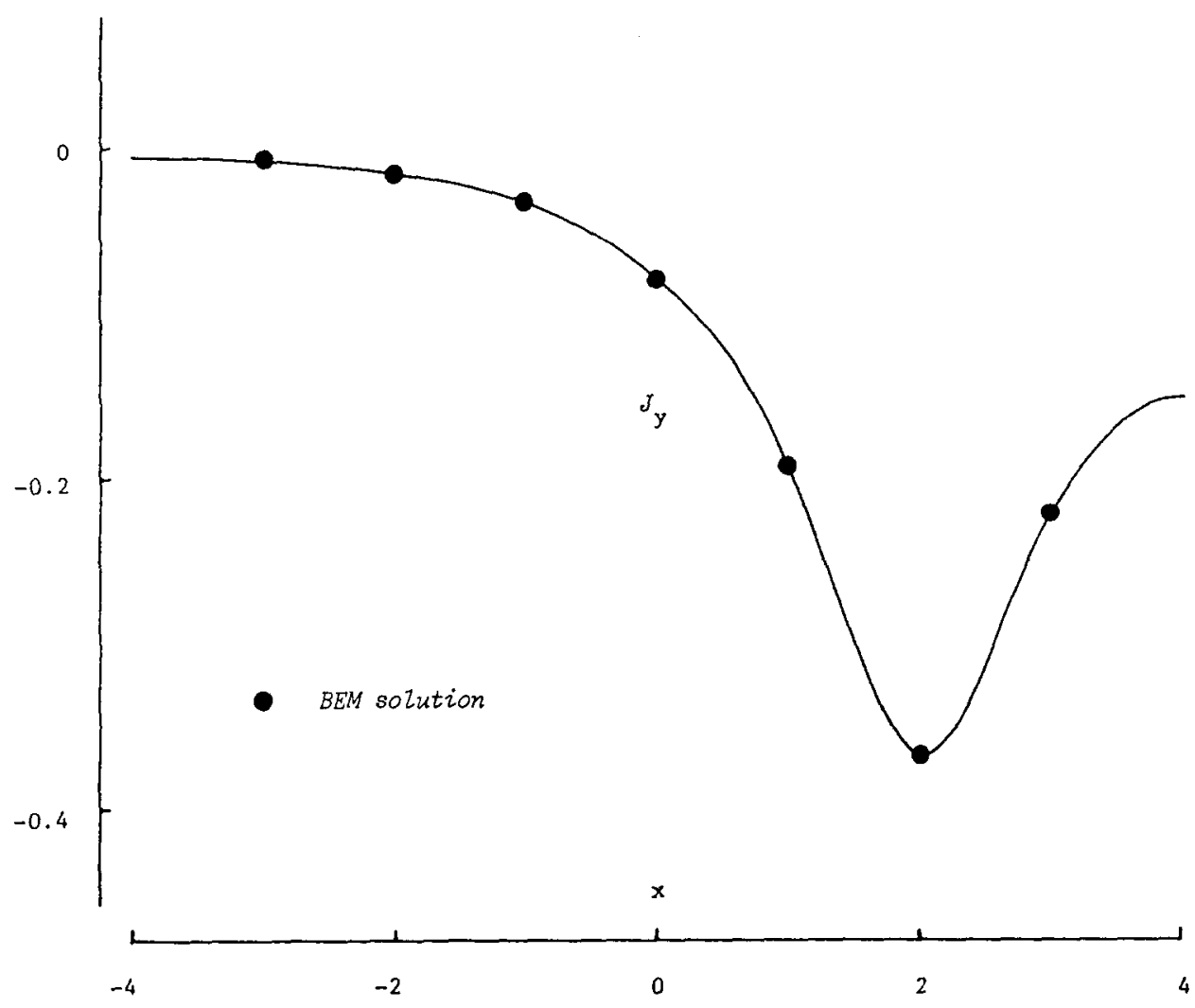

Fig. 8. Boundary-element solutions for the current density $J_{y}$ together with the analytical profile along the line of $y=0$. In the boundary element method, the whole-space scalar fundamental solution is used with analytical integrations for the Neumann problem of Fig. 7. 
An attempt is then made to satisfy (53) at the nodes of boundary elements. Once the surface potential is approximately determined over each boundary element, the potential at the field point $\boldsymbol{x}$ (not on $\Gamma$ ) can be computed also by using (53).

We practice analytical integrations in computing (53). Figs. 8 and 9 show the computed current densities along the line of $y=0$ and $x=0$, respectively. Solutions on boundaries $\Gamma_{h}^{-}$ and $\Gamma_{g}^{+}$are depicted in Figs, 10 and 11 respectively. We further plot the numerical potential along $x=0$ in Fig. 12. All results are in good agreement with exact ones.

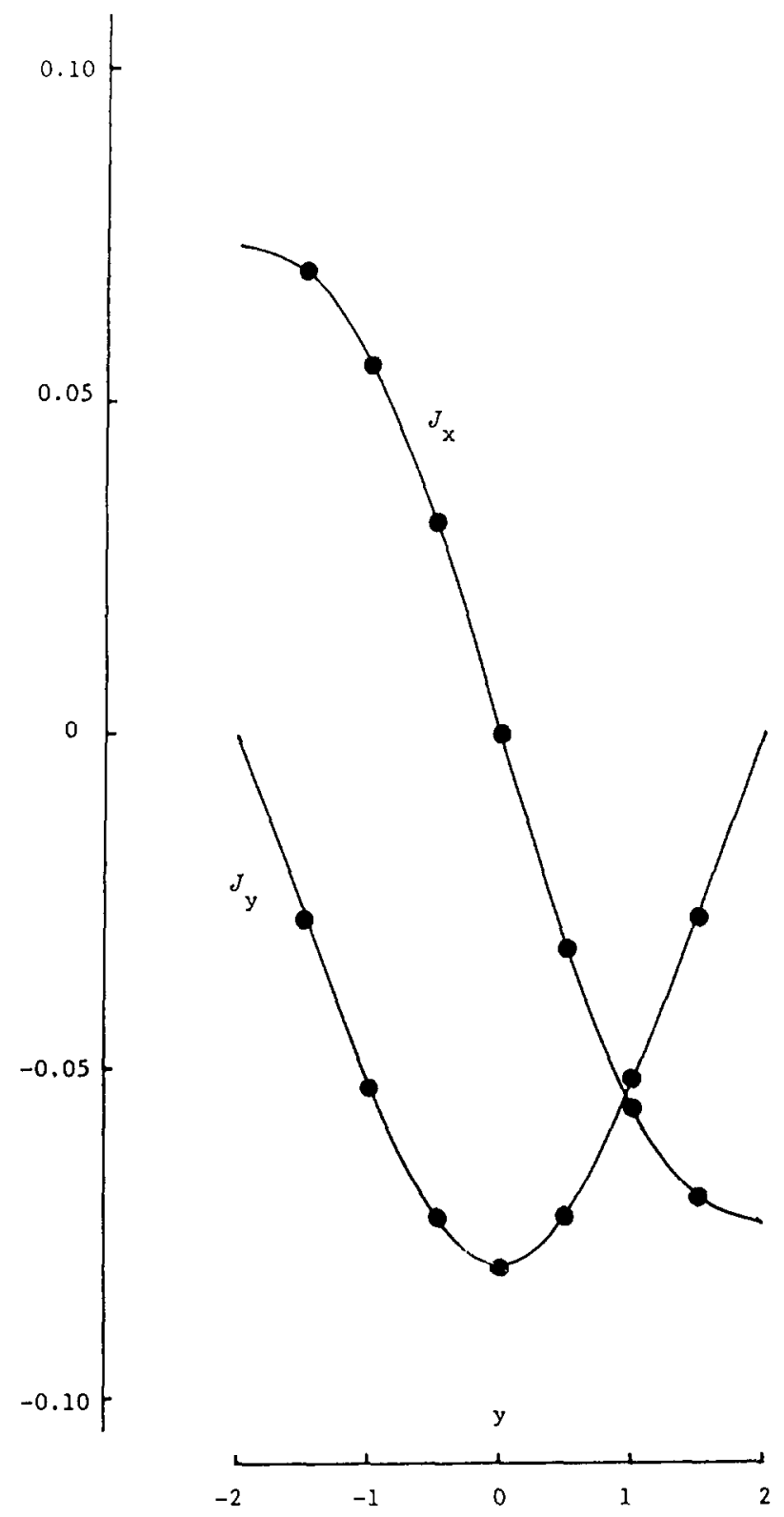

Fig. 9. Current densities $J_{x}$ and $J_{y}$ along the line of $x=0$ in the Neumann rectangle problem. The boundaryelement solutions are due to analytical integrations. 


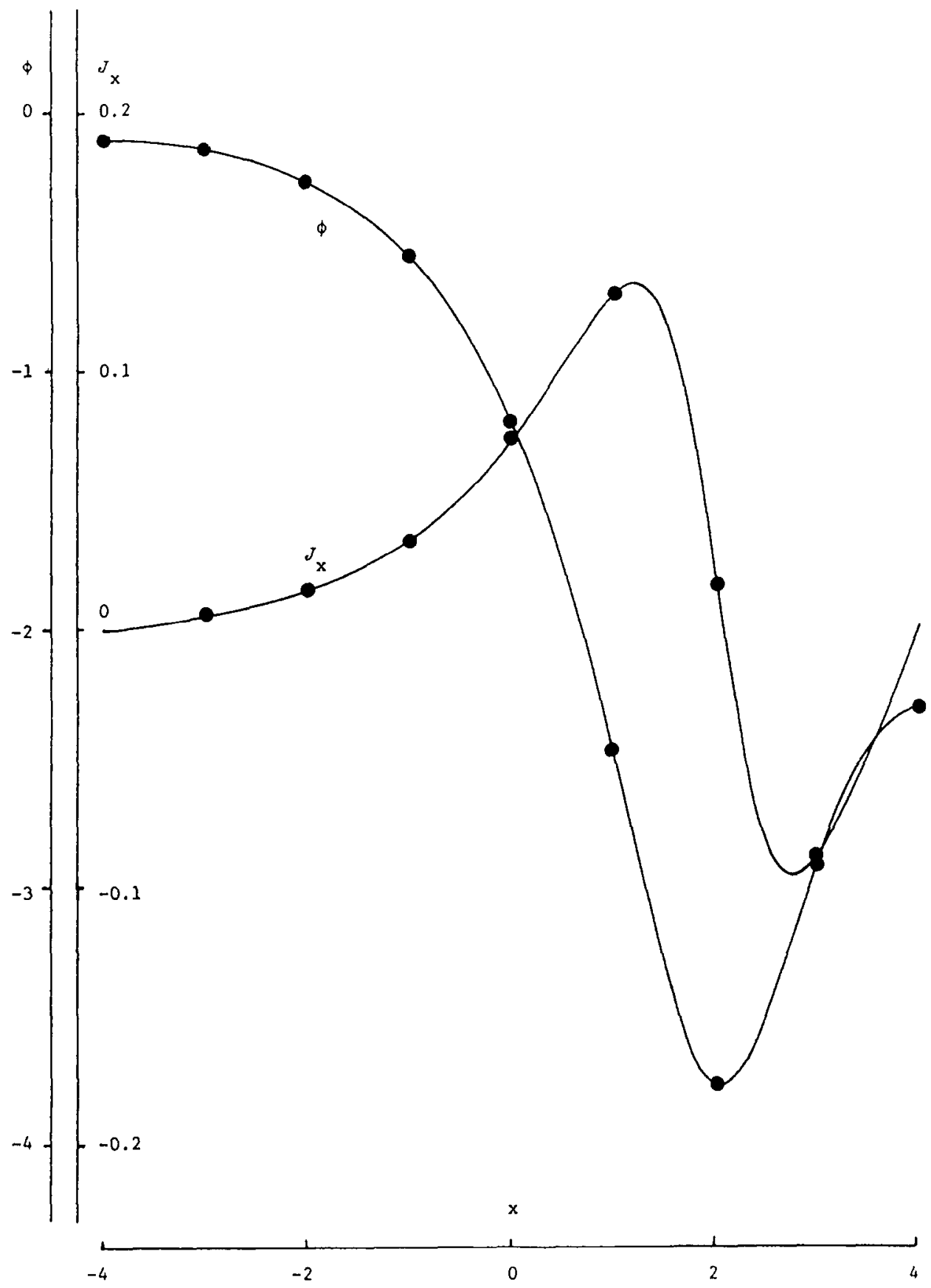

Fig. 10. Boundary-element solutions due to analytical integrations on the boundary $\Gamma_{h}^{-}$in the Neumann rectangle problem. 


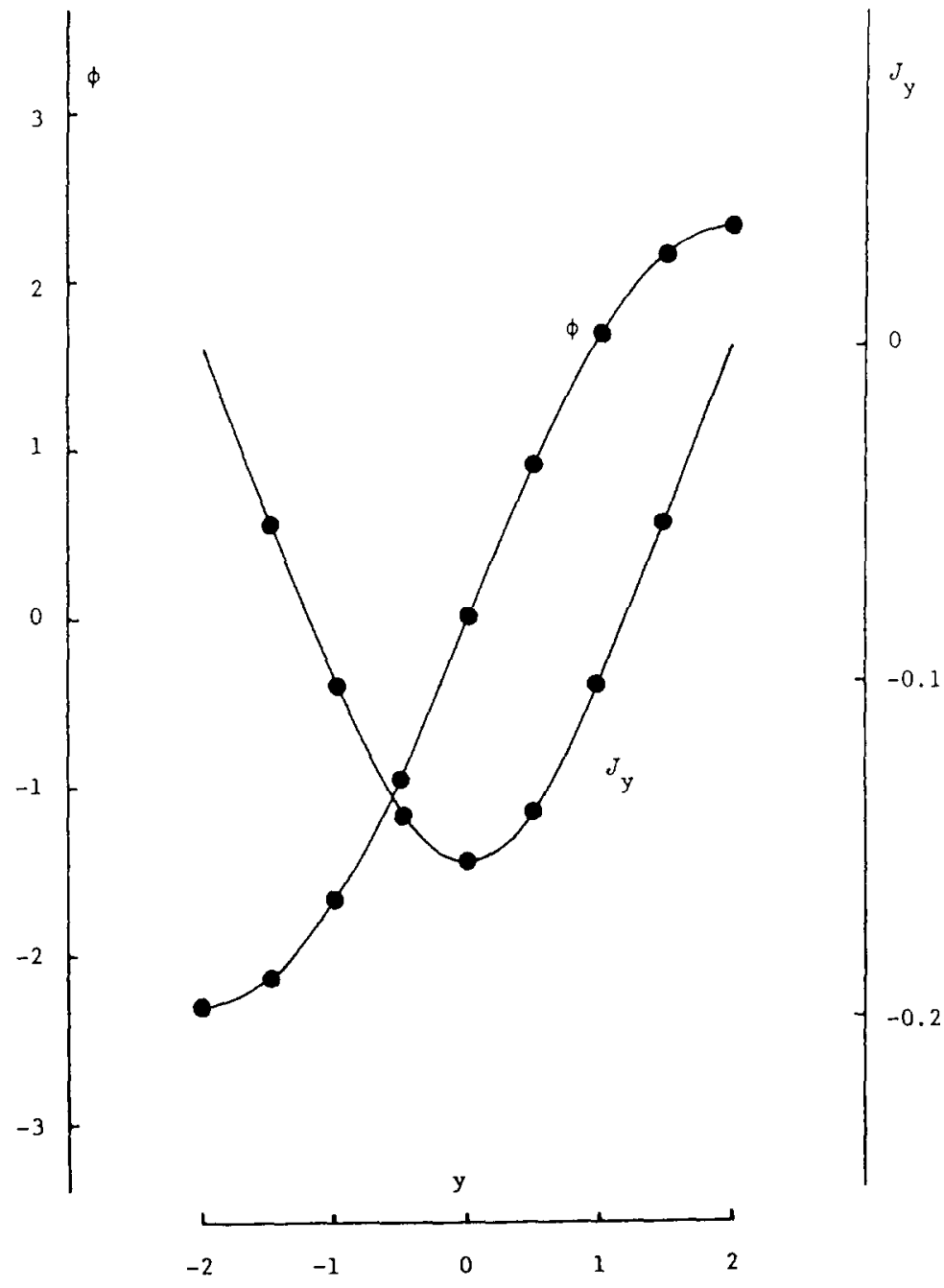

Fig. 11. Boundary-element solutions on $\Gamma_{g}^{+}$due to analytical integrations in the Neumann rectangle problem.

Boundary-element solutions due to 2-point Gauss quadrature are shown in Fig. 13 along the line of $x=0$. Numerical results are strongly influenced by the accuracy of integrations. Within the vicinity of the boundary surface, a nose-dive is further taken into half of the numerical potential value on the surface. This is because the numerical quadrature cannot treat a term which yields theoretically the appropriate singularity constant. In the case of analytical integrations, on the other hand, such a term can automatically be considered, and numerical solutions are everywhere adequate, see Fig. 12.

Suppose that another fundamental solution is adopted in (53) so that its normal derivative takes zero on some part $\Gamma-\hat{\Gamma}$. Then the numerical potential can be smooth near the part even by approximate integrations. Evidently analytical integrations are not always possible, and the use of more complicated fundamental solution should thus be investigated in the boundaryelement approach. We emphasize that all analytical solutions presented in this paper can be applied to the boundary integral techniques as typical fundamental solutions. For a Neumann 


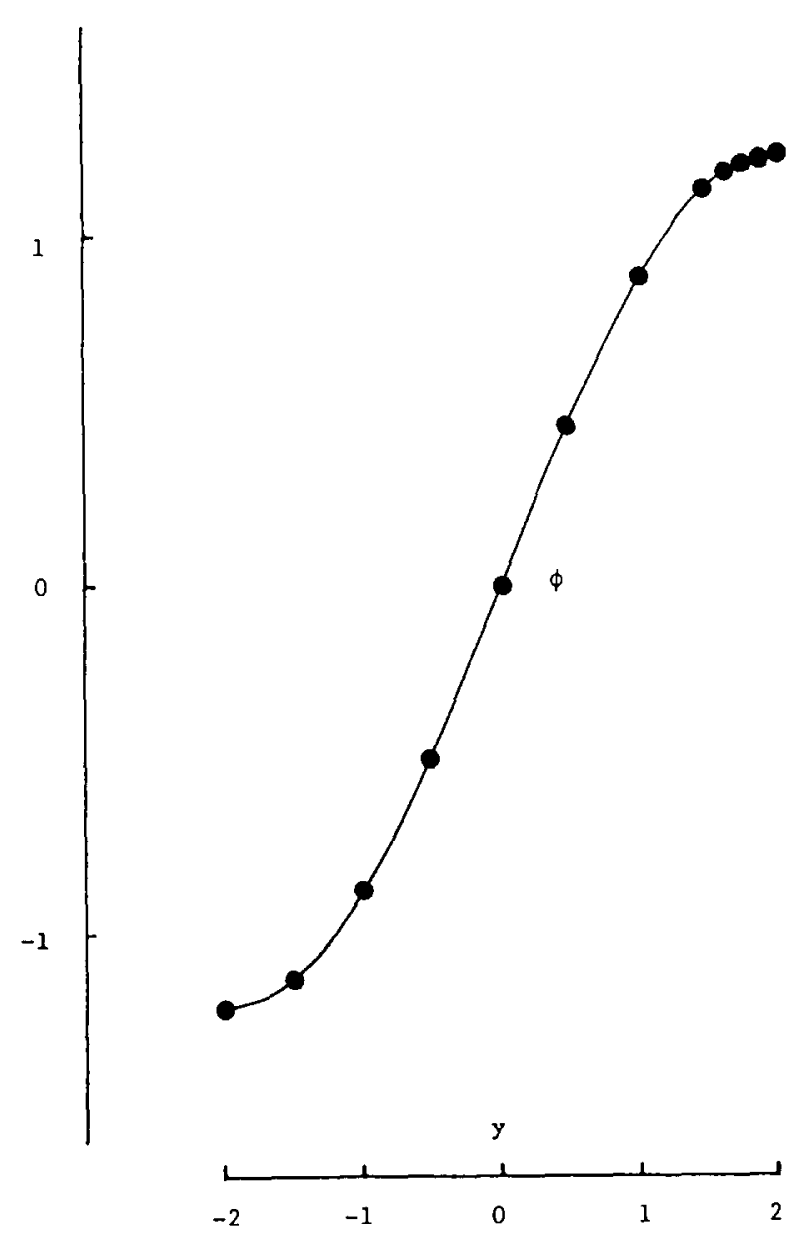

Fig. 12. Boundary-element solutions for the potential along the line of $x=0$ due to the analytical integrations in the Neumann rectangle problem.
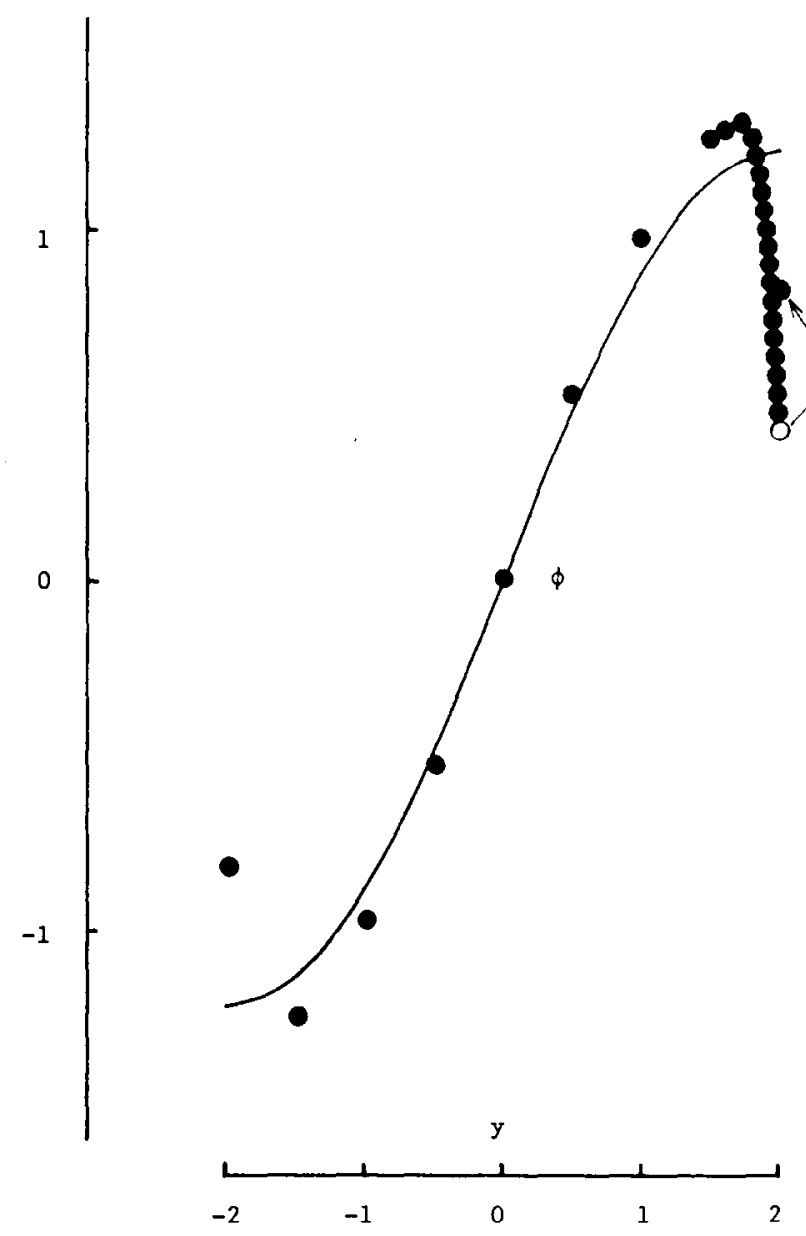

Fig. 13. Boundary-element solutions for the potential along the line of $x=0$ due to Gauss numerical quadratures in the Neumann rectangle problem.

problem within the domain $\Omega$ of Fig. 14, we show $\hat{\Gamma}$ associated with typical fundamental solutions in Table 5.

REMARK 8.1. On the smooth boundary, the tangential current density can be computed by using the differentiated form of (53). Here the singularity constant of $\frac{1}{2}$ should be considered. No other components are obtained because of singularities which cannot be avoided in this form. In order to compute those values, we must introduce more complicated fundamental solutions.

REMARK 8.2. In the Dirichlet problem, (53) on $\Gamma$ is written as

$$
0=\phi_{\mathrm{P}}(\boldsymbol{x})+\int_{\Gamma} \sigma \phi^{*}(\tilde{\boldsymbol{x}}, \boldsymbol{x}) \tilde{\boldsymbol{n}} \cdot \tilde{\nabla} \phi(\tilde{\boldsymbol{x}}) \mathrm{d} \Gamma, \quad x \in \Gamma
$$




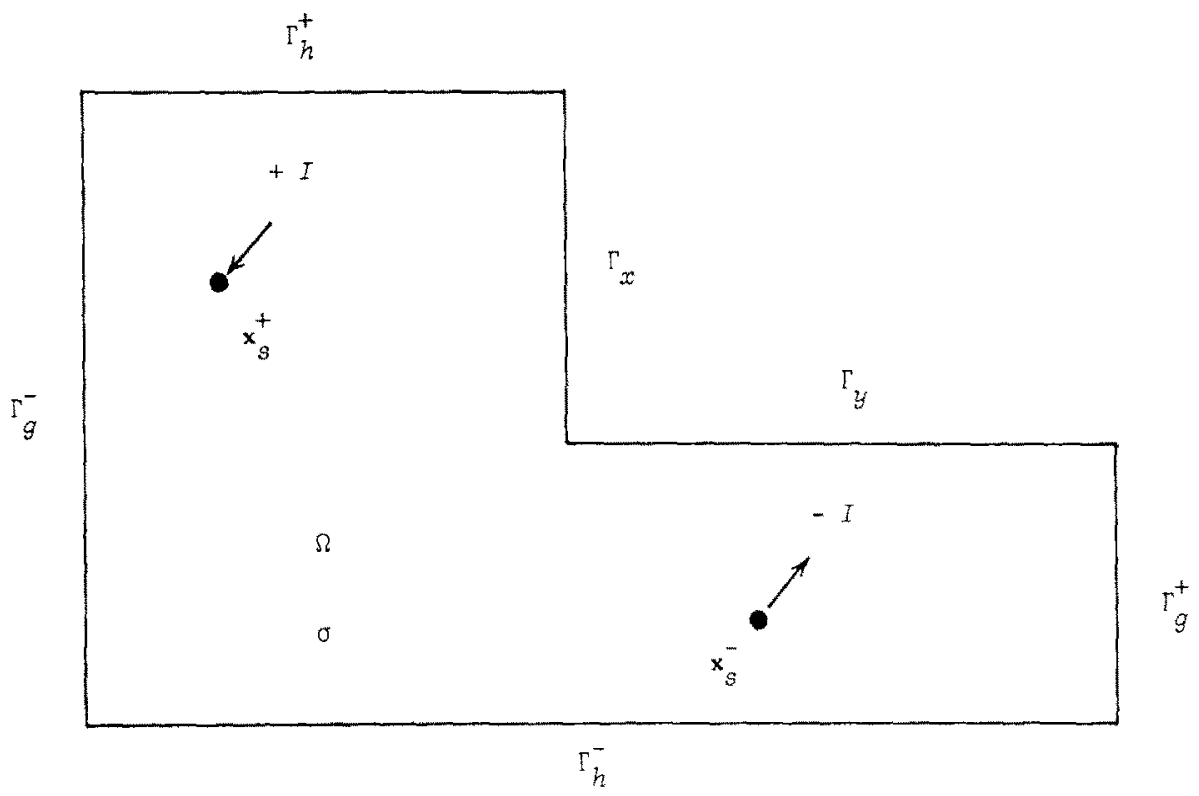

Fig. 14. Geometry of the typical Neumann problem.

Table 5

Typical fundamental solutions with $\hat{\Gamma}$ to be taken into considerations in the integral equation (53) for the Neumann problem of Fig. 14

\begin{tabular}{|c|c|}
\hline$\phi^{*}(\bar{x}, x)$ & $\hat{\Gamma}$ where $\tilde{\boldsymbol{n}} \cdot \tilde{\nabla} \phi^{*}(\tilde{\boldsymbol{x}}, \boldsymbol{x}) \neq 0$ \\
\hline$-\frac{1}{2 \pi \sigma} \ln R_{0}(\tilde{x}, x)$ & $\Gamma_{x}, \Gamma_{y}, \Gamma_{g}^{ \pm}, \Gamma_{h}^{ \pm}$ \\
\hline$-\frac{1}{2 \pi \sigma} \ln \left[R_{0}(\tilde{x}, x) / R_{1}(\tilde{x}, x)\right]$ & $\Gamma_{x}, \Gamma_{y}, \Gamma_{\mathrm{g}}^{ \pm}, \Gamma_{h}^{-}$ \\
\hline$-\frac{1}{4 \pi \sigma} \ln [F(\tilde{x}, \tilde{y}, x, y, h) G(\tilde{x}, \tilde{y}, x, y, h)]$ & $\Gamma_{x}, \Gamma_{y}, \Gamma_{z}^{ \pm}$ \\
\hline $\begin{array}{l}-\frac{1}{4 \pi \sigma} \ln [F(\tilde{x}, \tilde{y}, x, y, h) G(\tilde{x}, \tilde{y}, x, y, h) \\
\quad \cdot F(\tilde{x}, \tilde{y}, 2 g-x, y, h) G(\tilde{x}, \tilde{y}, 2 g-x, y, h)]\end{array}$ & $\Gamma_{x}, \Gamma_{y}, \Gamma_{\mathrm{x}}$ \\
\hline$-\frac{1}{4 \pi \sigma} \psi(\tilde{x}, x)$ & $\Gamma_{x}, \Gamma_{y}$ \\
\hline
\end{tabular}

Such a Fredholm integral equation of the first kind with respect to $\tilde{\boldsymbol{n}} \cdot \tilde{\nabla} \phi$, in general, results in somewhat unstable numerical solutions. Hence, the other type of the boundary integral techniques should be established in the Dirichlet problems, see [10].

\section{Finite-element solutions}

We then solve the Neumann rectangle problem by the finite element method. The rectangle of Fig. 7 is divided into 32 square elements of size $1 \times 1$, and computed values are plotted in Fig. 


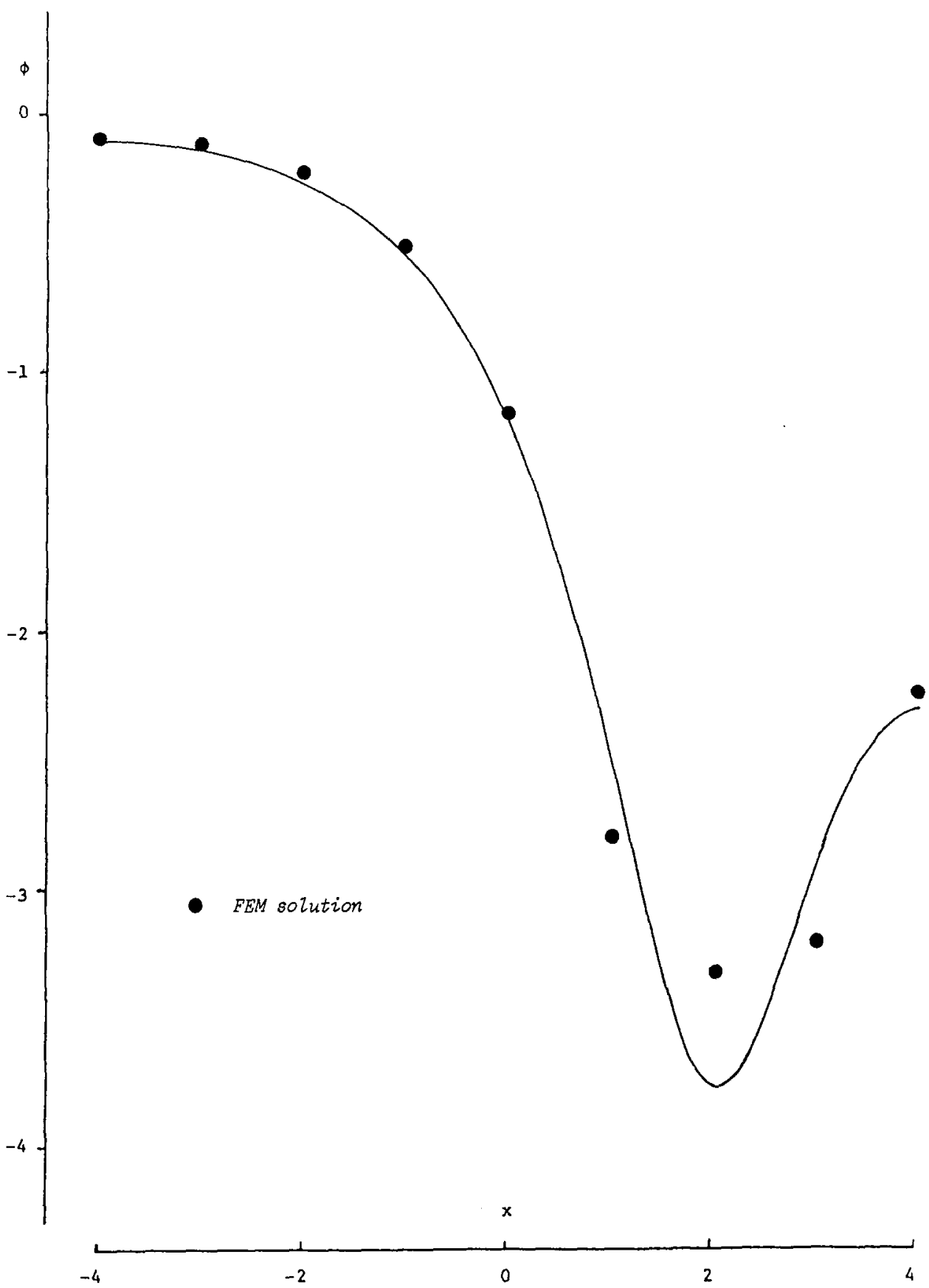

Fig. 15. Finite-element solutions along the line of $y=0$ in the Neumann rectangle problem. 


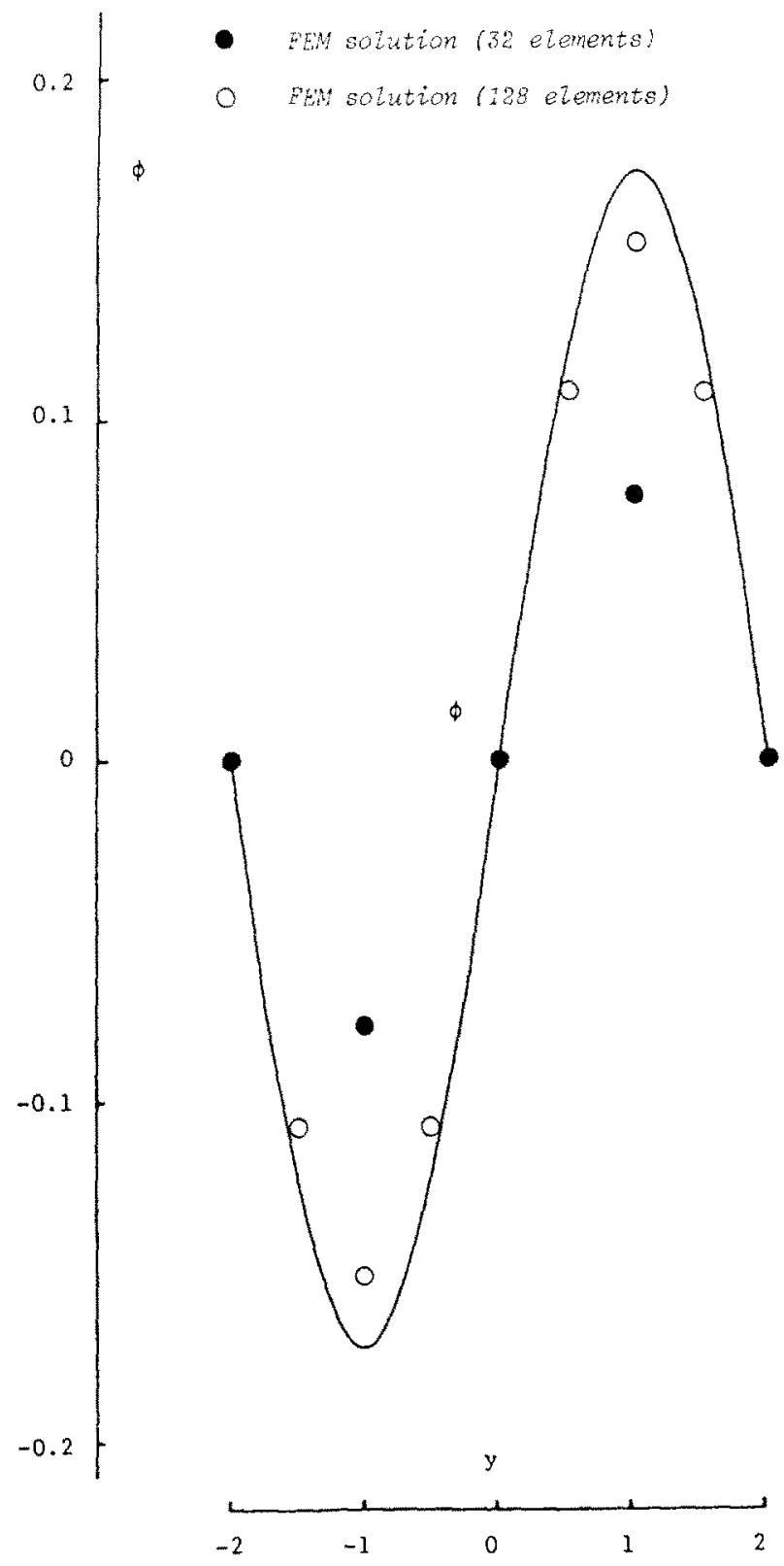

Fig. 16. Finite-element solutions along the line of $x=0$ in the Dirichlet rectangle problem.

15 along the line of $x=0$. Here the piecewise bilinear trial function is assumed over each finite element.

In the same way, the dirichlet rectangle problem is also solved and the finite-element solutions are depicted in Fig. 16. In the same figure, we show the improved results due to 128 squares of size $\frac{1}{2} \times \frac{1}{2}$.

Finite-element solutions are thus somewhat less accurate in comparison to boundaryelement solutions. We may have to investigate the self-adaptive convergence further in finite element applications. 


\section{Concluding remarks}

We have developed analytical solutions of electrical potential in the rectangular domain as well as in the infinite fissure, which are applicable to the boundary integral techniques as fundamental solutions. Alternative expressions may be obtained, for example by using Fourier series. We emphasize, however, that our solutions converge quitc rapidly which is of great importance in boundary-element applications. Examples are then presented to examine the finite-element and boundary-element solutions. Here the simple domain is preferable since no extra approximation techniques such as curvilinear mapping or infinite-domain interpolations are needed.

Our solutions may also play an important role in developing analytical solutions for linear elastic materials as well as for incompressible viscous flows within the simple rectangular domain.

\section{Acknowledgment}

The authors are grateful to Prof. N. Takenaka of Nihon University, for his comments and suggestions. Thanks are also due to Prof. J.T. Oden of the University of Texas at Austin, for his communication.

\section{References}

[1] C.A. Brebbia, The Boundary Element Method for Engineer (Pentech, London, 1978).

[2] M. Okabe, A boundary integral approach in the geoelectrical cavity prospecting. Comput. Meths. Appl. Mech. Engrg. 29 (1981) 297-311.

[3] J.H. Argyris, Triangular elements with linearly varying strain for the matrix displacement method, J. Roy. Aero. Soc. 69 (1965) 711-713.

[4] J.H. Argyris, K.E. Buck, I. Fried, G. Mareczek and D.W. Scharpf, Some ncw clements for matrix displacement methods, Proc. of 2nd Conf. on Matrix Methods in Structural Mechanics, Air Force Inst. Tech. Wright Patterson Air Force Base, OH, 1968.

[5] O.C. Zienkiewicz, The Finite Element Method (McGraw-Hill, London, 3rd ed., 1977).

[6] M. Okabe, Y. Yamada and I. Nishiguchi, Reconsideration of rectangular Lagrange families with hierarchyranking bases, Comput. Meths. Appl. Mech. Engrg. 23 (1980) 369-390.

[7] M. Okabe and N. Kikuchi, Some general Lagrange interpolations over simplex finite elements with reference to derivative singularities, Comput. Meths. Appl. Mech. Engrg. 28 (1981) 1-25.

[8] M. Okabe, Singularity constants and positioning constants in Poisson's equation with a point source of unit intensity, Comput. Meths. Appl. Mech. Engrg. 25 (1981) 287-297.

[9] R.V. Churchill, Complex Variables and Applications (McGraw-Hill, New York, 1960).

[10] M. Okabe, Boundary element method for the arbitrary inhomogeneities problem in electrical prospecting, Geophys. Prospect. 29 (1981) 39-59. 